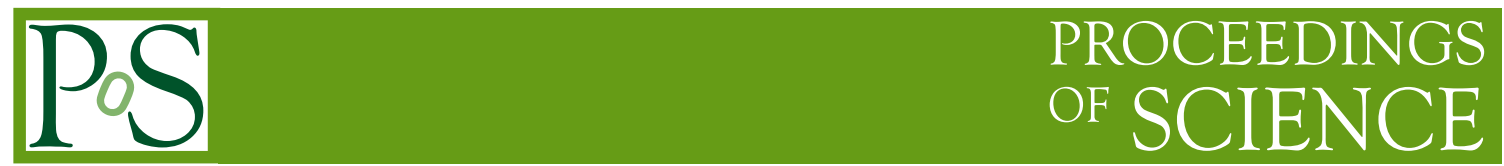

\title{
Recurrence relations in the large space-time dimension limit
}

\author{
P.A.Baikov ${ }^{* \dagger}$ \\ Moscow State University Skobeltsyn Institute of Nuclear Physics \\ E-mail: baikov@theory.sinp.msu.ru.
}

The technique, recently used for calculations of the 4- and 5-loop QCD radiative corrections, is reformulated in terms of the expansion of the recurrence relations in the large space-time dimension limit.

8th International Symposium on Radiative Corrections (RADCOR)

October 1-5 2007

Florence, Italy

\footnotetext{
*Speaker.

${ }^{\dagger}$ Supported by INTAS grant 03-51-4007 and RFBR grant 05-02-17645.
} 
Recurrence relations [1]] are widely used tool for calculations in perturbative quantum filed theory. Integration by parts provide us with some difference relations

$$
R\left(I^{-}, I^{+}, d\right) F\left(n_{1}, \ldots, n_{k}, d\right)=0
$$

where $F\left(n_{1}, \ldots, n_{k}, d\right)$ are Feynman integrals with propagators raised to integer (maybe negative) powers $n_{i}$ and $d$ is space-time dimension. In many cases these relations can be rebuilt in the recurrence form, thus allowing reduction procedure with the result

$$
F(\underline{n}, d)=C_{1}(\underline{n}, d) F_{1}+\ldots+C_{k}(\underline{n}, d) F_{k}
$$

where $F_{i}$ are "basic" or "irreducible" integrals with fixed values of $n_{i}$. Lets assume that we know how to calculate universal set $F_{i}$ and concentrate on $C_{i}$ which consist of specific for some physical problem information.

Unfortunately, direct reduction mentioned above not always possible. Rebuilding in recurrent form is nontrivial work in many-parametric case: hundreds of difficult sub-cases should be considered. Moreover, even constructed, reduction procedure involves long substitutions which can easily overflow any computer available.

The idea is to calculate $C_{i}$ directly, without reduction. Note that $C_{i}(\underline{n}, d)$ are rational in $d$ and obey the same difference relations:

$$
R\left(I^{-}, I^{+}\right) C_{i}(\underline{n}, d)=0
$$

with boundary conditions $C_{i}(\underline{n}, d)=0$ if $n_{l} \leq 0$ for at least one line of the basic integral associated with $C_{i}$. In the following we will call such associated lines or indexes "hard", and others "soft". Lets expand in $1 / d \rightarrow 0$ :

$$
\begin{gathered}
R\left(I^{-}, I^{+}\right)=R^{(0)}+\frac{1}{d} R^{(1)} \\
C_{i}=C_{i}^{(0)}+\frac{1}{d} C_{i}^{(1)}+\frac{1}{d^{2}} C_{i}^{(2)}+\ldots \\
0=R\left(I^{-}, I^{+}\right) C_{i} \quad \Rightarrow \quad \\
0=R^{(0)} C_{i}^{(0)} C_{i}^{(1)}+R^{(1)} C_{i}^{(0)} \\
\\
\quad \cdots \\
0=R^{(0)} C_{i}^{(k)}+R^{(1)} C_{i}^{(k-1)}
\end{gathered}
$$

The hope is that these equations are simpler so one can calculate sufficiently many coefficients and then reconstruct exact rational $d$ dependence.

Lets check it with 1-dimensional example:

$$
f_{n}=\int_{-\infty}^{\infty}\left(x^{2}+2 x+2\right)^{d} / x^{n} d x
$$




$$
n f_{n+1}=(d+1-n) f_{n}+(d+1-n / 2) f_{n-1}
$$

Direct reduction is trivial, but let's try $1 / d$ :

$$
\begin{aligned}
0 & =\underbrace{\left(f_{n}+f_{n-1}\right)}_{R^{(0)} f}+1 / d \underbrace{\left((1-n / 2) f_{n-1}+(1-n) f_{n}-n f_{n+1}\right)}_{R^{(1)} f} \\
0 & =R^{(0)} f^{(0)}=f_{n}^{(0)}+f_{n-1}^{(0)} \Rightarrow f_{n}^{(0)}=(-1)^{n} \\
0 & =R^{(0)} f^{(1)}+R^{(1)} f^{(0)} \\
& =f_{n}^{(1)}+f_{n-1}^{(1)}+n / 2(-1)^{n} \Rightarrow f_{n}^{(1)}=1 / 4\left(n^{2}+n\right)(-1)^{n}
\end{aligned}
$$

For simplicity we skip contributions which reflect $f_{n} \rightarrow c(d) f_{n}$ uncertainty. In this example leading order equation involves only $I^{-}$operators (no explicit $n_{i}$ coefficients). Such equations can be solved in multi-dimensional case:

$$
R\left(I^{-}\right) C_{i}=0 \quad \Rightarrow \quad C_{i}(\underline{n})=\Pi_{a} r_{a}^{-n_{a}}, \quad \text { where } R\left(r_{a}\right)=0 .
$$

Indeed: $R\left(I_{a}^{-}\right) C_{i}=R\left(r_{a}\right) C_{i}=0$. Shortly speaking equations without explicit $n_{i}$ coefficients simpler then general ones like algebraic equations simpler then differential ones.

Lets now consider Feynman integrals

$$
\begin{gathered}
F(\underline{n}, d)=\int d^{d} p_{1} . . d^{d} p_{L} /\left(E_{1}^{n_{1}} \cdots E_{a}^{n_{a}}\right), \\
E_{a}=A_{a}^{i k}\left(p_{i} p_{k}\right)+m_{a}^{2} .
\end{gathered}
$$

Integration by parts

$$
\begin{gathered}
0=\int d^{d} p_{1} . . d^{d} p_{L} \partial_{p_{i}}\left(p_{k} \cdots\right), \\
\partial_{p_{i}}\left(p_{k} \cdot\right)=d \delta_{k}^{i}+p_{k}\left(\partial_{p_{i}} \cdot\right)=d \delta_{k}^{i}+(A A)_{b}^{a} E_{a}\left(\partial_{E_{b}} \cdot\right), \\
(A A)_{b}^{a}=2\left(A^{-1}\right)_{k l}^{a} A_{b}^{l i}
\end{gathered}
$$

provide us with the difference equations

$$
0=d \delta_{k}^{i} F+(A A)_{b}^{a} E_{a} \partial_{E_{b}} F .
$$

If we now define

$$
\begin{gathered}
0=d \delta_{k}^{i} F+\left(A A_{k}^{i}\right)_{b}^{a} E_{a} \partial_{E_{b}} F \\
R^{(0)} ? \quad R^{(1)} ?
\end{gathered}
$$

we will face with trivial solution only

$$
0=R^{(0)} C^{(0)}=\delta_{k}^{i} C_{n}^{(0)} \quad \Rightarrow \quad C_{n}^{(0)}=0 \quad ? ?
$$


In fact it means that there are no solutions in the simple form $C_{i}=C_{i}^{(0)}+\frac{1}{d} C_{i}^{(1)}+\ldots$. Indeed, $C_{k} \approx d^{-S(\underline{n})}$, where $S(\underline{n})$ is the sum of the "hard" $n_{i}$.

In practice we apply $1 / d$ expansion to the sub-case when all "hard" $n_{i}=1\left(n_{i}>1\right.$ can be reduced to $n_{i}=1$ by direct recursion [1, 3, (4). Lets modify our relations in order to decouple "hard" and "soft" indexes. For that consider integration by parts with additional insertion in numerator (it is equivalent to recombination of original relations with polynomial in $E_{a}$ factors):

$$
0=\int d^{d} p_{1} . . d^{d} p_{L} \partial_{p_{i}}\left(p_{k} \Pi^{i k}\left(E_{a}\right) \cdots\right) .
$$

With some polynomials $\Pi^{i k}$ we come to diagonalized relations

$$
\begin{aligned}
& 0=\partial_{E_{a}}(P(E) F)-(d-L-1) / 2\left(\partial_{E_{a}} P(E)\right) F, \\
& R^{(1)} \quad \text { large } \quad R^{(0)}
\end{aligned}
$$

where $P(E)=\operatorname{det}_{i k}\left(p_{i} p_{k}\left(E_{a}\right)\right)$. Equations with "hard" $\partial_{E_{a}}$ can be used for $n_{a} \rightarrow 1$ reduction. In the equations with "soft" $\partial_{E_{a}}$ we can set "hard" $E_{a}=0$ (these operators shift $n_{a}$ from 1 to 0 , and by definition $C_{i}=0$ if at least one "hard" $n_{i}$ is non-positive). So for "soft" $\partial_{E_{a}}$ for leading order in $1 / d \rightarrow 0$ limit we got non-trivial equations

$$
0=R^{(0)} F^{(0)}=\left(\partial_{E_{a}} P(E)\right) F^{(0)} .
$$

In principle one can proceed further as with toy 1-dimensional example, but we found more efficient to calculate $C_{i}^{(k)}(\underline{n})$ by expanding auxiliary integrals in $1 / d \rightarrow 0$ limit $[4$

$$
C_{i}^{(k)}(\underline{n})=\int d x_{1} . . d x_{a} /\left(x_{1}^{n_{1}} . . x_{a}^{n_{a}}\right) P(\underline{x})^{(d-L-1) / 2} .
$$

In $1 / d \rightarrow 0$ they expand to Gaussian type integrals

$$
\int d x_{1} . . d x_{a} x_{1}^{k_{1}} . . x_{a}^{k_{a}} \exp \left(-A^{i k} x_{i} x_{k}\right)
$$

which can be efficiently evaluated in gamma-functions.

Let us now briefly discuss possible applications of the method. The ideal case is massless 0-scale problems: $1 / d$ coefficients are pure numbers and the set of basic integrals are relatively small. As the result it was successfully applied to the calculation of the variety of 4- and 5-loop quantities in QCD [5].

One can also try 1-mass 0 -scale problems, but in this case number of basic integrals increases, which means that more contributions should be calculated and total efforts (setup + CPU demands) became comparable to other approaches [6]; nevertheless cross-checks still will be useful. Finally, the method potentially applicable to the reduction of multi-scale integrals, but in this case the calculation of basic integrals became very complicated (being the key problem).

To summarize, the expansion of the difference equations for Feynman integrals in the limit of large $d$ allows to solve them in systematic way. The mathematical complexity of the original equations is partially transformed to the additional computer resources demands, but the variety of physically relevant 4- and 5-loop problems are reachable. 


\section{References}

[1] K. G. Chetyrkin and F. V. Tkachov, Integration By Parts: The Algorithm To Calculate Beta Functions In 4 Loops, Nucl. Phys. B 192 (1981) 159.

F. V. Tkachov, A Theorem On Analytical Calculability Of Four Loop Renormalization Group Functions, Phys. Lett. B 100 (1981) 65.

[2] P. A. Baikov, Explicit solutions of the 3-loop vacuum integral recurrence relations, Phys. Lett. B 385 (1996) 404 [arXiv:hep-ph/9603267].

[3] P. A. Baikov and M. Steinhauser, Three-loop vacuum integrals in FORM and REDUCE, Comput. Phys. Commun. 115 (1998) 161 [arXiv:hep-ph/9802429].

[4] P. A. Baikov, A practical criterion of irreducibility of multi-loop Feynman integrals, Phys. Lett. B 634 (2006) 325 [arXiv:hep-ph/0507053].

[5] P. A. Baikov and K. G. Chetyrkin, Top Quark mediated Higgs decay into hadrons to order alpha(s)**5, Phys. Rev. Lett. 97, 061803 (2006) [arXiv:hep-ph/0604194].

P. A. Baikov, K. G. Chetyrkin and J. H. Kuhn, Scalar correlator at O(alpha(s)**4), Higgs decay into b-quarks and bounds on the light quark masses, Phys. Rev. Lett. 96, 012003 (2006) [arXiv:hep-ph/0511063].

P. A. Baikov, K. G. Chetyrkin and J. H. Kuhn, Strange quark mass from tau lepton decays with O(alpha(s**3)) accuracy, Phys. Rev. Lett. 95, 012003 (2005) [arXiv:hep-ph/0412350].

P. A. Baikov, K. G. Chetyrkin and J. H. Kuhn, Five-loop vacuum polarization in $p Q C D: O(m(q) * 2$ alpha(s)**4 $n(f) * * 2)$ contribution, Phys. Lett. B 559, 245 (2003) [arXiv:hep-ph/0212303].

P. A. Baikov, K. G. Chetyrkin and J. H. Kuhn, Towards order alpha(s)**4 accuracy in tau decays, Phys. Rev. D 67, 074026 (2003) [arXiv:hep-ph/0212299].

P. A. Baikov, K. G. Chetyrkin and J. H. Kuhn, The cross section of e+e-annihilation into hadrons of order alpha $(s) * * 4 n(f) * * 2$ in perturbative $Q C D$, Phys. Rev. Lett. 88, 012001 (2002) [arXiv:hep-ph/0108197].

[6] S. Laporta and E. Remiddi, The analytical value of the electron ( $g$-2) at order alpha 3 in QED, Phys. Lett. B 379 (1996) 283 [arXiv:hep-ph/9602417].

P. Mastrolia and E. Remiddi, Precise evaluation of the electron ( $g$-2) at four loops: The algebraic way, Nucl. Phys. Proc. Suppl. 89 (2000) 76.

A. V. Smirnov and V. A. Smirnov, Applying Groebner bases to solve reduction problems for Feynman integrals, JHEP 0601, 001 (2006) [arXiv:hep-lat/0509187].

A. V. Smirnov, An algorithm to construct Groebner bases for solving integration by parts relations, JHEP 0604 (2006) 026 [arXiv:hep-ph/0602078].

A. V. Smirnov and V. A. Smirnov, On the reduction of Feynman integrals to master integrals, arXiv:0707.3993 [hep-ph]. 